\title{
Basic design rules of unit cells for additive manufactured lattice structures
}

\author{
Julia Kessler ${ }^{1, *}$, Nicolae Balc ${ }^{2}$, Andreas Gebhardt ${ }^{1}$, and Karim Abbas ${ }^{1}$ \\ ${ }^{1}$ University of Applied Sciences Aachen, Faculty of Mechanical Engineering and Mechatronics, Goe- \\ thestr. 1, 52064 Aachen, Germany \\ ${ }^{2}$ Technical University of Cluj-Napoca, Department of Manufacturing Engineering, B-dul Muncii, \\ 103-105, 400641 Cluj-Napoca, Romania
}

\begin{abstract}
Additive Manufacturing is a cutting-edge technology for the production of complex, functional, filigree and topology-optimized components. The growing demand for high-tech light-weight structures increasingly requires support in the design process of fine lattice structures. However, due to the high information density regarding lattice structures, which is based on many scientific research studies, the effective design of a specifically product-oriented lattice structure is made more difficult. The focus of this work is to provide a user-friendly information basis for the user or constructor. Therefore, this scientific work serves to show the first basic rules for the design of simple unit cells for additive lattice structures. The approach is based on the evaluation of the limiting manufacturing factors, which can be reduced both to the minimum and maximum angle and length, as well as to the cross-sectional geometry. The analysis reveals a considerable extent to the cross-sectional geometries and their welding pattern in various quality forms. The results obtained form the basis for the development of complex functional unit cells. However, the construction of a unit cell is only a part of a lattice structure, so additional factors must be added to the unit cell assembly.
\end{abstract}

\section{Introduction}

Additive Manufacturing (AM) is the world-wide standard for the manufacturing technology of layered processes known as Rapid Prototyping in the late 1980s. Where the conventional production technologies reach their limits, additive production shows its strengths. The key benefits of this technology are faster product development and recovery, better product characteristics and significant cost savings. In order to tap the potential, however, it is necessary to have experience, knowledge and the right tools about these technologies. The additive technologies offer various advantages, especially in the production of highly complex components with integrated functionality or lightweight structures. This applies in particular in the area of the layer-wise construction of metal components by means of Selective Laser Melting. Specifically, the higher complexity of a component through functional integration, internal structures or a topology-optimized design can be implemented without additional costs with SLM. The SLM process allows the manufacture of components with integrated hollow or lattice structures that significantly reduce the 
volume to be built. But an existing problem lies in the handling and the direct integration of various grid structures into an existing component. Especially the commercial user is often overstretched, as there are many scientific knowledge but no explicit procedure for the application of lattice structures within the scope of the SLM production. Various software solutions exist on the market for the design of such lightweight structures within a component, such as Magics Structures or Autodesk Within. The commonality of these software solutions is that the structures can be multiplied by unit cells of uniform size in the component interior and can be adapted to mechanical loads, especially with Autodesk Within, by means of thickness optimization. Empirical studies and simulations using the finite element method (FEM) have shown that the size and shape of the unit cells influence the mechanical properties of the lattice structure. The only deviation from the regular unit cell is currently made to the transition area to the outer surfaces of the hollow body by rotating or distorting the unit cells there [1].

The most important element of a lattice structure is the unit cell, which may have different properties. In addition to the packing density of a cell, the single-cell cell shape and its webs are particularly decisive. The current state of the art shows that mainly the additive production of periodic lattice structures has been investigated. Extensively, cubic lattice structures were investigated by different researches, especially by Olaf Rheme. At first, under the consideration of the essential production restrictions of the SLM, cubic lattice structure types are analysed and classified by crystallography. It is clear to say that rarely other non-cubic structures, such as Honeycomb structures, Voronoi structures and topology optimization structures are analysed. At present, more researchers and users are discovering the potential of generatively fabricated lattice structures. For the SLM, periodic lattice structures are suitable, due to the targeted geometric influence. Open cell types are necessary to remove the excess powder material. Various production processes, such as, for example, the fine casting, are used for producing grid structures. In contrast to other manufacturing processes, SLM can produce virtually any desired structures arranged in space and is thus also suitable for topology optimization of components [2].

The evaluation of lattice structures requires a differentiated approach of several influencing factors. These include first and foremost the process parameters, machine specific influencing variables and the geometric properties of the to be printed parts.

All these three main focuses are in a corresponding constellation and depend on each other. The machine specific influencing variables are constant and they describe the values that are mainly defined by the plant technology. These for example include the integrated laser scanning system or rather the laser itself. Properties like the scanning speed or the laser power are based on the plant technology but finally are not constant. These parameters can be adjusted and controlled manually with an integrated machine software. Adjustable parameters like the bevor mentioned ones, belong to the process specific influencing factors. Furthermore, an important value is defined by the construction of the product. The main SLM process, the material and the functional principle of the SLM plant define and set the process boundaries as well as the construction boundaries. All adjustments, values and parameters form the basis for the product quality and thus the resulting properties of the generated product. The process specific influencing factors primarily influence the quality of the generated parts. First of all, the laser and the original material are the essential values within the SLM process. The interaction between metal powder and laser decide about the precision and the boundaries of the to be generated parts. [2, 3, 4]

\section{State of the art and current research}

Process based pores that appear in the struts reduce the mechanical properties and the actual aimed quality of structures. Concerning this, Chunlei Qin et al. investigated the relation- 
ship between the laser beam and the molten pool. The influences of the laser-power and the scanning speed were the focus of this investigation. The evaluation was performed with the help of microscopes and high-speed-imaging. The results showed that the laser-power has a decisive influence on the strut-diameter and that an interaction of the laser beam and the molten lead to a violent molten pool. This violent interaction causes the generation of pores and bad surface-roughness. [5]

One basic work in this field of research titled "Cellular Design for Laser Freeform Fabrication" was presented by Olaf Rehme [2]. His work contributes to understand the mechanical properties of lattice structures made by SLM. After a detailed description of all process constraints and boundary conditions for the manufacture of SLM lattice structures, he makes a theoretical analysis to comprise the examination of structural hypotheses for isotropic cellular materials before a generalized theory is developed for anisotropic SLM lattice structures. He evaluates different cell types such as polyhedral, cubic and rhombic cells towards their producibility. His scientific work gives information about extensive experimental evaluation of test specimens of the properties of SLM solids and lattice structures by SLM. He shows that SLM lattice structures allow a very high degree of design freedom in comparison to conventional cellular materials. The most important finding of his thesis is that power laws, which are normally used to describe mechanical properties of cellular materials, break down above a certain critical relative density. He identified one specific cell tyy (f2ccz) which excels other cell types by far and is overall best performing due to its high specific strength, high specific energy absorption and high specific stiffness when loaded under compressive stresses, especially in z-direction. Besides he gives two examples for implementation of the SLM lattice structures into applications, e.g. medical endoprostheses in order to prove how innovative products can be furnished with new functionalities, such as faster ingrowth of bone tissue.

Chunze Yan, Liang Hao, Ahmed Hussein, Philippe Young, and David Raymont describe in their paper "Advanced lightweight 316L stainless steel cellular lattice structures fabricated via selective laser melting" [6] the manufacturability and performance of advanced and lightweight stainless steel cellular lattice structures fabricated via selective laser melting. A unique cell type called gyroid is designed to construct periodic lattice structures and utilize its curved cell surface as a self-sup-ported feature which avoids the building of support structures and reduces material waste and production time. The experimental set-up is about a cell size of $5 \mathrm{~mm}$, with a volume fraction for massive parts of $6,8,10,12$ and $15 \%$ and $9 \times$ specimens with the dimensions $25 \times 25 \times 15 \mathrm{~mm}$. The cellular lattice structures were made from a $316 \mathrm{~L}$ stainless steel powder with average particle size of $45 \pm 10 \mu \mathrm{m}$. The results of the compression properties describe that the worst orientation lattice structure offers higher mechanical properties than the normal orientation lattice structure although both of them have the same volume fraction of $15 \%$ and unit cell size of $5 \mathrm{~mm}$, indicating anisotropy of the gyroid cellular lattice structure. The Young's modulus of the worst orientation lattice structure is $302.57 \mathrm{MPa}$, which is $20.37 \%$ greater than that of the normal orientation lattice structure, $251.36 \mathrm{MPa}$, and the yield strength of the worst orientation lattice, $15.53 \mathrm{MPa}$, is $7.78 \%$ higher compared with the yield strength of the normal orientation lattice structure, $14.41 \mathrm{MPa}$.

Based on these and many other investigations, the application of lattice structures as mechanical design elements requires to take into account the final geometry of the struts, the material to be integrated into the engineering design and the material properties obtained from metal printing process. For this reason, the aim of the work is to create a basis for the Commercial User to facilitate the handling of lattice structures and their integration into special components. In addition to the various types of grid structures, their mechanical properties must also be analysed in detail in order to enable the user to select a corresponding grid structure individually and according to his selection criteria. 


\section{Research approach}

First, a pre-study was carried out to limit the process parameters and to evaluate the material used and the associated possibilities, such as the powder grain size, which has a decisive influence on the maximum resolution and the smallest possible construction elements. Because of the fact that the fundament of every periodic lattice structure are the struts for this purpose, it was analyzed which geometries of the struts can be realized. They determine the value of the mechanical properties, the performance and finally the quality. Concerning the struts conclusions have been made in previous researches. Topics included the feasibility and the reached quality. Most of the time struts with a circular shape are set as the main shape and used for the investigation. Just a few use elliptical or different shapes. $[6,7]$ Thus the influence of the cross-sectional shape on the loading resistance is rarely taken into consideration. But basic researches are essential and create the necessity to take a closer look on this topic. At the beginning, it is important to know what causes a limitation on the generation of struts. The focus is on the thinnest to be reached struts and the building accuracy of the cross-sectional area. All in all, the main aim is a feasibility study. Properties of five different cross sectional shapes are examined: a circular-, an elliptical-, a square-, a triangular- and a rhombus shape. On several platforms, a matrix of struts is placed with the bevor mentioned shapes (Figure 1).

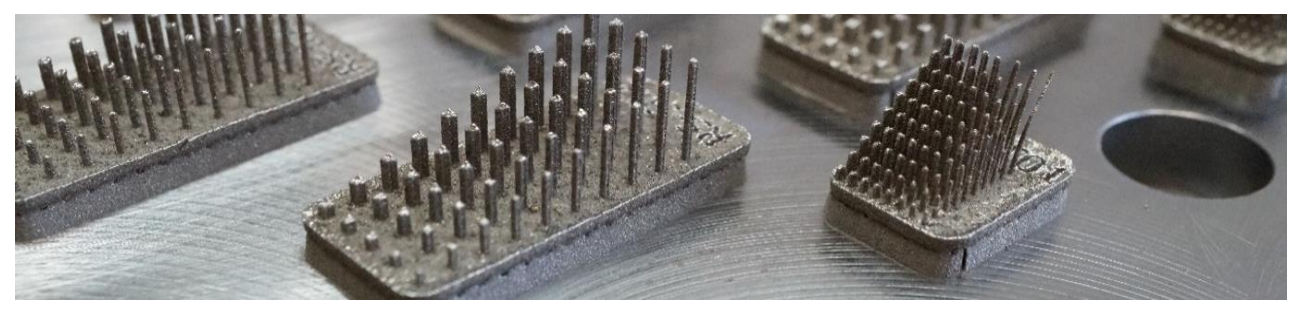

Fig. 1. Matrix of different struts and geometries.

These are designed with a constant increasing cross sectional area in $\mathrm{x}$-direction and a length variation in $\mathrm{y}$-direction up to $10 \mathrm{~mm}$. The source for each platform is a digital $3 \mathrm{D}$ model. For a better evaluation of the structural limits, the experimental set up was split into three different constructive starting points. At first a reference surface (RS) was set to find out what the limits are, regarding to the cross sectional are. Furthermore, the limits of the outlines of the shapes were evaluated with setting a collective greatest diagonal line or width (RO). The last specimen pool had also a predefined cross-sectional area, which was based on the area of a circular shape (RC), because most of the lattice structures consist of circular struts. Within this investigation three different initial constructive approaches have been compared. Therefore, the optical evaluation with a microscope was essential. The greatest strut width was measured and subsequently the average and the deviation were calculated. The aim was the comparison of the set point and the average value. To analyse the most important focuses of the results the quality of the struts is additionally consulted. The evaluation of the struts with a circular cross section shows some characteristics. It is noticeable that struts with a nominal diameter smaller than $0,15 \mathrm{~mm}$ cannot be generated. The smallest reached diameter aggregates towards an average of $0,201 \mathrm{~mm}$, the constrained set point is $0,16 \mathrm{~mm}$, which almost is a deviation of $25 \%$. Striking is that every strut within the set point range of $0,15 \mathrm{~mm}$ up to $0,25 \mathrm{~mm}$, does not overwrite an average value of $0,2 \mathrm{~mm}$ up to $0,25 \mathrm{~mm}$. Every reached diameter overwrites the actual to be reached design value. In all three experimental set ups it is noticeable that from a value of $0,35 \mathrm{~mm}$ a turning point appears. This turning point describes the moment from which the deviations shift into the negative values and thus the average values are smaller than the actual set point. Comparing 
the conditions of the manufactured struts a characteristic is recognizable, that describes the above-mentioned phenomenon. It is obvious that struts up to a diameter of $0,25 \mathrm{~mm}$ are just towered welding beads. They don't appear to be cylindrical outlines und consist of irregular spherical shapes. A selection of the cross-sections to be examined could be made over the weld pattern of the struts. The determination of the smallest $(0,4 \mathrm{~mm} 2)$, medium $(0,7 \mathrm{~mm} 2)$, and largest $(1,0 \mathrm{~mm} 2)$, cross-section area was carried out.

\section{Experimental Set-Up}

The above-described pre-study was necessary in order to determine the feasibility of the specimens with different geometries of the bridge and to show which sizes and geometries the process can produce. A basic research based on this aim is to create an evaluation of the boundaries for the design, based on the interplay of geometry and process properties.

In this series of experiments, the aim is to define significant values for the construction of the unit cell and its struts, both through different angular variations as well as different lengths and three selected cross-sectional areas of the struts.

For the experiment, a total of 33 specimens with different geometries circle, ellipse, square, triangle, rhombus, were tested. Each sample was build up at different angles in $10^{\circ}$ degree steps. As can be seen in Figure 2, a sample body consists of a $5 \mathrm{~mm}$ wide sheet and ten struts arranged thereon. The length as well as the cross-sectional area remain constant for every angular change. Beyond the five geometries, three different cross-sections 0.4 $\mathrm{mm}^{2}, 0.7 \mathrm{~mm}^{2}$ and $1.0 \mathrm{~mm}^{2}$ are tested, which also result from the preliminary test. In addition, the lengths are varied. Generated are $5 \mathrm{~mm}$ long struts as well as $10 \mathrm{~mm}$ long struts. The bridge-to-base connection is a flat plane and not spherical (see picture 3 with a purple mark).

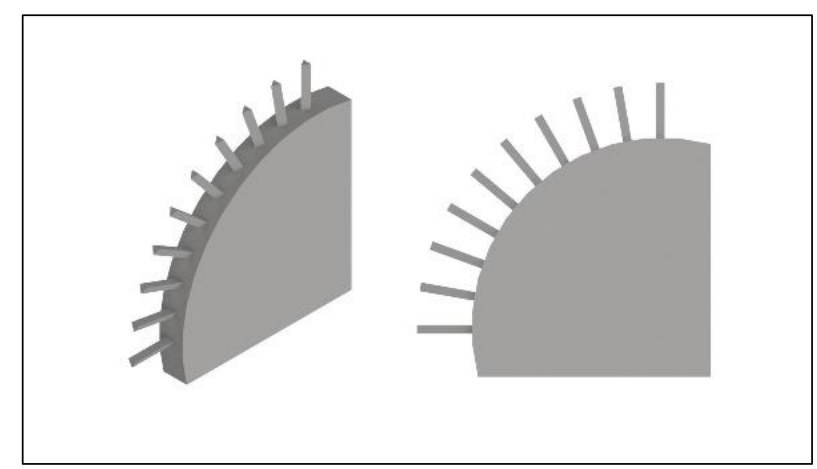

Fig. 2. Sample with different angles in $10^{\circ}$ degree steps.

The samples are manufactured by SLM with a M1 Cusing machine (Concept Laser). All components are printed with constant process parameters, which have proven to be the optimum in the previous tests. However, this will not be discussed further. The most important parameters are defined by a laser power of $180 \mathrm{~W}$, a scanning speed of $1450 \mathrm{~mm} / \mathrm{s}$ for contours and $1000 \mathrm{~m} / \mathrm{s}$ for surface exposure. The layer thickness is $45 \mu \mathrm{m}$ and a grain size distribution of $30-60 \mu \mathrm{m}$. 


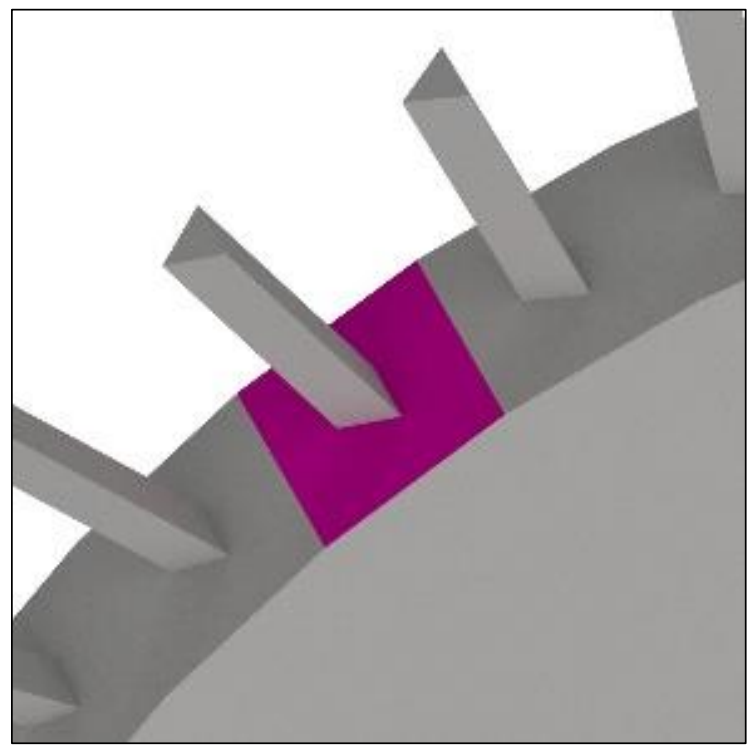

Fig. 3. Bridge-to-base connection as a flat plane.

The analysis of the samples is carried out optically with the aid of a microscope. The struts are microscopy and photographed from different perspectives in order to be subsequently evaluated. The analysis and evaluation of the samples gives information about the minimum and maximum angle as well as the length to be realized. In addition, the quality of the bridges and the realized geometries can be assessed.

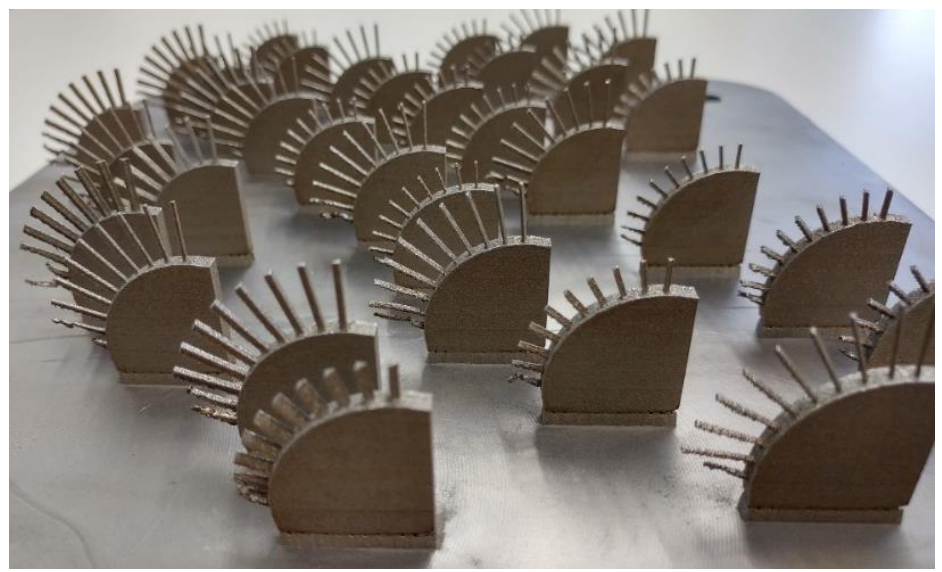

Fig. 4. Samples on the building platform of the SLM machine.

\section{Results and discussion}

Since this is an optical evaluation, criteria have previously been defined which serve for a better assessment, which is used to evaluate all struts. One special focus was on the welding traces and welding beads. Here, the front and the lateral sides of the struts were considered.

The aim of the test series is to evaluate the boundaries and influences of different strut geometries on the generation of struts in different angles. As a result, struts are determined 
that are optimally defined according to the criteria and meet the requirements. The following table shows such an assessment and analysis.

Table 1. Protocol of the test series (example triangle, $5 \mathrm{~mm}$ strut length, $0,4 \mathrm{~mm} 2$ cross-sectional area).

\begin{tabular}{|c|c|c|c|c|c|c|c|c|c|}
\hline \multicolumn{10}{|c|}{ Protocol Microscopy angle samples } \\
\hline \multirow{2}{*}{\multicolumn{3}{|c|}{$\begin{array}{l}\text { Geometry } \\
\text { Triangle }\end{array}$}} & \multirow{2}{*}{$\begin{array}{c}\text { Cross-sectional area } \\
0,4 \mathrm{~mm}^{2}\end{array}$} & \multirow{2}{*}{\multicolumn{3}{|c|}{$\begin{array}{l}\text { Strut lenght } \\
5 \mathrm{~mm}\end{array}$}} & \multirow{4}{*}{\begin{tabular}{|c|}
$\begin{array}{l}\text { Welding beads } \\
\text { front apparent }\end{array}$ \\
indefinable \\
\end{tabular}} & \multirow{4}{*}{\begin{tabular}{|c|}
$\begin{array}{r}\text { Welding beads } \\
\text { coat apparent }\end{array}$ \\
indefinable \\
\end{tabular}} & \multirow{4}{*}{ Features } \\
\hline & & & & & & & & & \\
\hline Probe & Angle $\left({ }^{\circ}\right)$ & & Geometrie realized & \begin{tabular}{|c|} 
Alignment \\
geometry to the \\
building platform
\end{tabular} & $\begin{array}{c}\text { Strut } \\
\text { realized }\end{array}$ & Surface quality & & & \\
\hline 1 & 0 & & indefinable & a parallel zur BP & & indefinable & & & \\
\hline 2 & 10 & & indefinable & $\begin{array}{c}\text { a parallel to } \\
\text { building platform }\end{array}$ & & $\begin{array}{c}\text { hardly adherent } \\
\text { fragments, very many } \\
\text { adherent fragments }\end{array}$ & indefinable & medium & Blue discolored downhill \\
\hline 3 & 20 & & $\begin{array}{l}\text { approximately triangular, } \\
\text { strong deformation downhill }\end{array}$ & $\begin{array}{c}\text { a parallel to } \\
\text { building platform }\end{array}$ & $\mathrm{x}$ & $\begin{array}{c}\text { hardly adherent } \\
\text { fragments, very many } \\
\text { adherent fragments }\end{array}$ & strong & medium & $\begin{array}{l}\text { Orange/Blue discolored } \\
\text { downhill }\end{array}$ \\
\hline 4 & 30 & & $\begin{array}{l}\text { approximately triangular, } \\
\text { strong deformation downhill }\end{array}$ & \begin{tabular}{|c} 
a parallel to \\
building platform
\end{tabular} & $\mathrm{x}$ & $\begin{array}{c}\text { hardly adherent } \\
\text { fragments, very many } \\
\text { adherent fragments }\end{array}$ & strong & edium & $\begin{array}{l}\text { Nose at the corner uphill, } \\
\text { welding points at an edge } \\
\text { uphill }\end{array}$ \\
\hline 5 & 40 & & $\begin{array}{l}\text { approximately triangular, } \\
\text { strong deformation downhill }\end{array}$ & $\begin{array}{c}\text { a parallel to } \\
\text { building platform }\end{array}$ & $\mathrm{x}$ & $\begin{array}{c}\text { hardly adherent } \\
\text { fragments, many adherent } \\
\text { fragments downhill }\end{array}$ & strong & $\begin{array}{l}\text { medium at the } \\
\text { edges, light on } \\
\text { the surface }\end{array}$ & $\begin{array}{l}\text { Nose at the corner uphill, } \\
\text { welding points at an edge } \\
\text { uphill }\end{array}$ \\
\hline 6 & 50 & $\mathrm{x}$ & $\begin{array}{l}\text { Slight discontinuities, slight } \\
\text { deformation downhill }\end{array}$ & $\begin{array}{l}\text { a parallel to } \\
\text { building platform }\end{array}$ & $\mathrm{x}$ & $\begin{array}{l}\text { hardly adherent } \\
\text { fragments, some } \\
\text { adherent fragments }\end{array}$ & strong & $\begin{array}{l}\text { medium at the } \\
\text { edges, light on } \\
\text { the surface }\end{array}$ & $\begin{array}{l}\text { Welding points on an edge } \\
\text { uphill }\end{array}$ \\
\hline 7 & 60 & $x$ & $\begin{array}{l}\text { Slight discontinuities, less } \\
\text { stable downhill }\end{array}$ & $\begin{array}{l}\text { a parallel to } \\
\text { building platform }\end{array}$ & $x$ & $\begin{array}{c}\text { hardly adherent } \\
\text { fragments, less } \\
\text { adherent fragments } \\
\text { downhill }\end{array}$ & strong & $\begin{array}{l}\text { medium at the } \\
\text { edges, light on } \\
\text { the surface }\end{array}$ & $\begin{array}{c}\text { Nose at the corner uphill, } \\
\text { welding points at an edge } \\
\text { uphill, bridge slightly curved } \\
\text { upwards }\end{array}$ \\
\hline 8 & 70 & $x$ & $\begin{array}{l}\text { Slight discontinuities, less } \\
\text { stable downhill }\end{array}$ & $\begin{array}{l}\text { a parallel to } \\
\text { building platform }\end{array}$ & $\mathrm{x}$ & hardly adherent fragments & strong & $\begin{array}{l}\text { medium at the } \\
\text { edges, light on } \\
\text { the surface }\end{array}$ & $\begin{array}{l}\text { strut slightly curved } \\
\text { upwards }\end{array}$ \\
\hline 9 & 80 & $\mathrm{x}$ & Minimal discontinuities & $\begin{array}{c}\text { a parallel to } \\
\text { building platform }\end{array}$ & $x$ & normal & strong & $\begin{array}{l}\text { light on the } \\
\text { surface }\end{array}$ & Nose at the corner uphill \\
\hline 10 & 90 & $\mathrm{x}$ & Minimal discontinuities & $\begin{array}{c}\text { a parallel to } \\
\text { building platform }\end{array}$ & $x$ & normal & strong & $\begin{array}{l}\text { light on the } \\
\text { surface }\end{array}$ & $\begin{array}{l}\text { On the forehead slightly } \\
\text { discolored }\end{array}$ \\
\hline
\end{tabular}

The analysis shows recurring abnormalities in all geometries, which show deviations to the models constructed. For all test specimens, the horizontal ridge is not fully generated. However, only a partial segment of the strut is produced, whereas the major part is deformed and distorted. The cross-section of the partial segment increases toward the base of the whole probe. This is comparable with struts with a $10^{\circ}$-building direction.

Table 2. Total evaluation of test series.

\begin{tabular}{|c|c|l|l|l|l|}
\hline \multicolumn{1}{|c|}{ Strut length } & $\underline{\mathbf{5 m m}}$ & & & & \\
\hline $\begin{array}{l}\text { cross-section area } \\
(\mathbf{m m 2})\end{array}$ & Triangle & Ellipse & Circle & Rhombus & Square \\
\hline 0.4 & $50^{\circ}-90^{\circ}$ & $50^{\circ}-90^{\circ}$ & $50^{\circ}-90^{\circ}$ & $60^{\circ}-90^{\circ}$ & $50^{\circ}-90^{\circ}$ \\
\hline 0.7 & $30^{\circ}-90^{\circ}$ & $60^{\circ}-90^{\circ}$ & $50^{\circ}-90^{\circ}$ & $50^{\circ}-90^{\circ}$ & $50^{\circ}-90^{\circ}$ \\
\hline 1.0 & $20^{\circ}-90^{\circ}$ & $30^{\circ}-90^{\circ}$ & $30^{\circ}-90^{\circ}$ & $40^{\circ}-90^{\circ}$ & $60^{\circ}-90^{\circ}$ \\
\hline$\underline{\text { Strut length }}$ & $\underline{\mathbf{1 0 m m}^{\circ}}$ & & & & \\
\hline $\mathbf{( m m 2 )}$ & $\mathbf{T r i a n g l e}$ & Ellipse & Circle & Rhombus & Square \\
\hline 0.4 & $50^{\circ}-90^{\circ}$ & $60^{\circ}-90^{\circ}$ & $50^{\circ}-90^{\circ}$ & $60^{\circ}-90^{\circ}$ & $50^{\circ}-90^{\circ}$ \\
\hline 0.7 & $30^{\circ}-90^{\circ}$ & $60^{\circ}-90^{\circ}$ & $50^{\circ}-90^{\circ}$ & $50^{\circ}-90^{\circ}$ & $50^{\circ}-90^{\circ}$ \\
\hline 1.0 & $30^{\circ}-90^{\circ}$ & $20^{\circ}-90^{\circ}$ & $30^{\circ}-90^{\circ}$ & $40^{\circ}-90^{\circ}$ & $60^{\circ}-90^{\circ}$ \\
\hline
\end{tabular}

Both for the horizontal and $10^{\circ}$-struts, significant oxidation is to be found on all areas which are directed towards the building platform. This discoloration only decreases at fur- 
ther larger angles. The unrealized subrange also decreases. Table 2 shows the results for the individual geometries and the corresponding fully realized struts with respect to the angle.

A further conspicuity shows that the actual geometry is very stretched or compressed at small angles of $20^{\circ}-50^{\circ}$ in all structures and does not correspond to the original model. This is illustrated in Figure 5. Along with the stretching and compression, an enlarged crosssectional area of the geometries can also be seen. In this case, the enlargement of the crosssection decreases to the extent up to the vertical strut is also shown in Figure 5 (top actual state - lower target state).

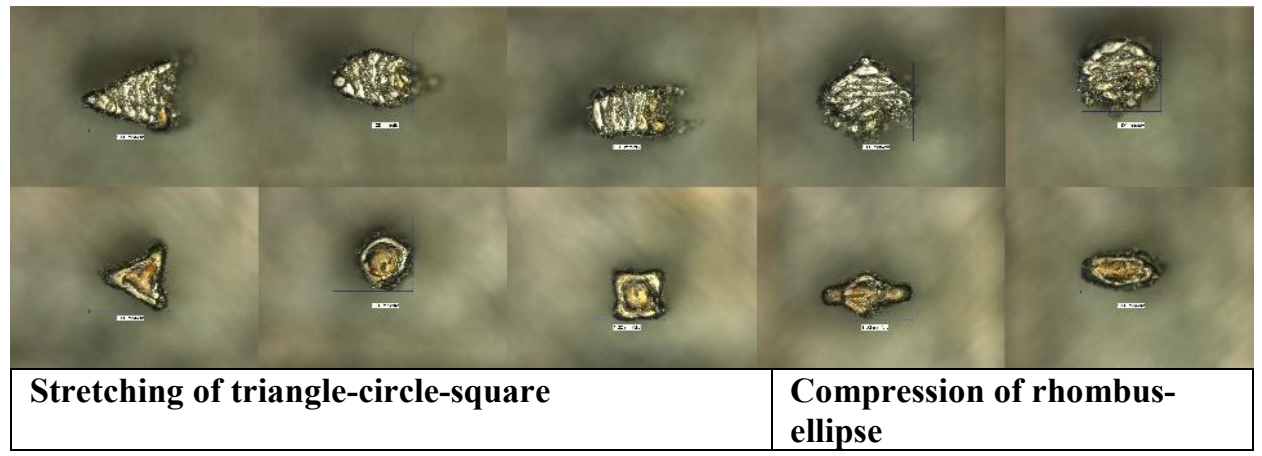

Fig. 5. Stretching of triangle, circle and square struts and Compression of rhombus and ellipse struts.

A conspicuousness in the welding traces is associated with the not fully generated struts. In the case of struts with visible deformation (pointing to the construction platform), two special areas are shown within the strut. These are similar for all geometries. These regions can be subdivided into welding traces which are located within the printing plane and are parallel to each other, as well as welding traces which are pointing towards the building platform and which are warped or deformed. This can be seen in Figure 6. However, this effect of bagging decreases with increasing angular size.

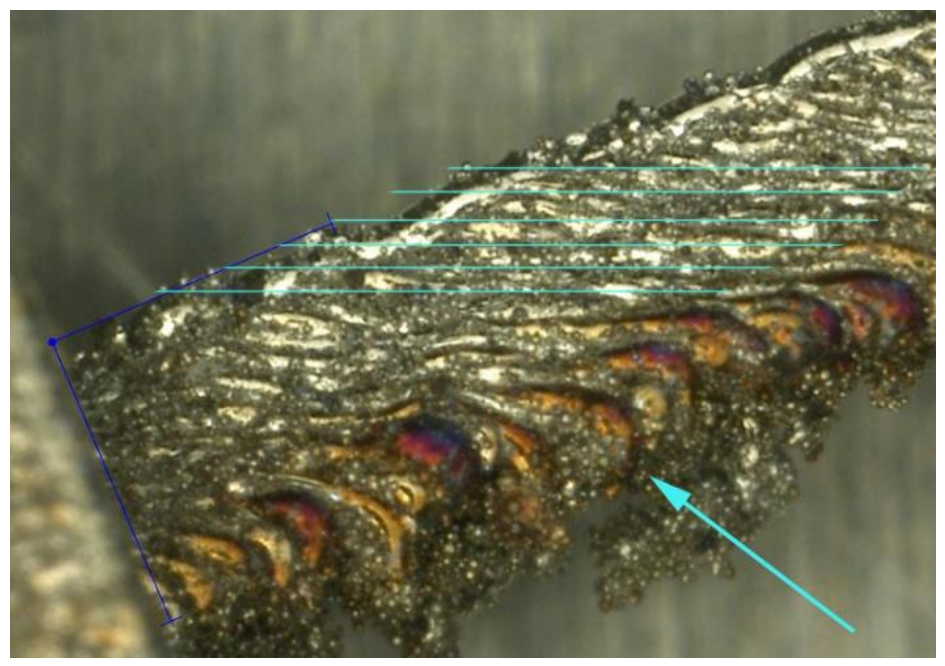

Fig. 6. Strut with welding traces.

The geometries are different basic geometries, but they are comparable in some respects, for example the triangle and square geometries. Both geometries are generated with a flat side pointing towards the building platform. There are similarities as well as differences in the comparison. Viewing the $20^{\circ}$-strut (Figure 7, third column) shows that the tri- 
angle geometry is strongly deformed and a partial segment is missing, but a part is generated so far that the welding layers and the plane are visible. On the other hand, in the case of the square, the strut is slightly warped and a larger sub-segment is missing. As a result of the geometry itself, the cross-section of the square is constant over the planes, while the cross-section of the triangle becomes smaller and tapers. The result for the process itself is that in the case of an advanced printing process, either constant thermal energy is induced or the thermally induced energy decreases with decreasing cross-section and thus less welding traces.

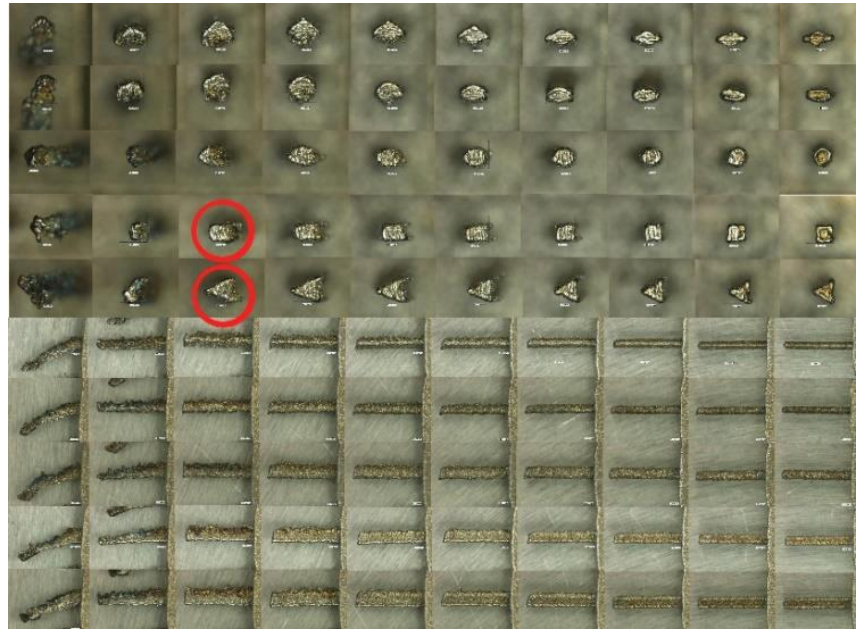

Fig. 7. Overview of all geometries and different angels (above: front side below: lateral sides of the struts).

A crucial question at the beginning of this study was if there are significant contour geometries that are significantly better than others at certain angles? But the research showed that it is only to a certain extent that struts with different geometries are better represented.

A further result of this survey is that the warping causes layers to lie in overlying planes and are exposed several times, resulting in clumping and tearing off the structure, what causes the welding process and oxidation.

The first assumption was that the strut length has a decisive effect on the geometry to be realized as a function of the cross-sectional area and the corresponding angles. The reason for this is the thermal distortion, since unsupported struts tend to deform at different angles. This assumption could not be confirmed except for minor deviations in the case of triangle and ellipse (deviation of $10^{\circ}$ ). Conclusion and outlook

The second assumption was that the generation of struts is decisively determined by the geometry and its cross-sectional areas. The assumption could be confirmed partly by means of the experimental series, however, it was found that only minor deviations could be analyzed when comparing the 0.4 series to the 0.7 series as well as the deviations of the 0.7 series to the 1.0 series. This leads to the exclusion of the 0.7 series for further experiments.

\section{Conclusion and outlook}

The aim of the investigations discussed in this paper was to develop a basis for a guideline for the design of simple unit cells for additive lattice structures. The main focus was on the evaluation of which geometry could be generated with which cross-section and angle. All geometries can be realized with a build-up angle of $20^{\circ}$, some with clear contours, the others with better contours with increasing build-up angle. Below the $20^{\circ}$, the struts are not 
very poor and have been realized with great deformations. It is striking that the trial triangle and ellipse have achieved very good results in the lower range (angles of $20^{\circ}-40^{\circ}$ ) compared to the other three geometries a possible reason for the triangle geometry could be the change of the temperature gradient due to the geometry. Due to the geometry itself, e.g. While the cross section of the triangle becomes smaller and tapers as the square crosses across the planes.

Further mechanical tests, such as the tensile test, should now provide information about the mechanical properties of these five different web geometries. After evaluating the tensile tests, a selection of the geometries which provide the best mechanical characteristic values is to be carried out. With these geometries, the newly developed unit cells are then constructed for the construction of individual lattice structures. These different lattice structure types are deposited with mechanical characteristics and will be main part of the guideline. The feasibility of geometries is not sufficient for the construction of a single cell, so the mechanical properties have to be evaluated. The mechanical tests include, but are not limited to, tensile/compression test, fatigue test, three-point bending test and torsion. These results will determine the decision criteria for the commercial user.

Most of the scientific work has concerned itself with cubic unit cells, and with the geometries of the individual bridges rather than with the geometry of the entire unit cell. This survey ensures that both complex polygon structures and cubic cells are possible. The aim of this research work is to generate a matrix with 12 different lattice structure types with both cubic and polygonal unit cells for the possibility of user-friendly selection of grid structures to allow the commercial user the possibility of user-friendly selection of grid structures for integration into certain components.

The guideline for the implementation of grid structures in additive manufactured components should include the essential steps: Analysis of the component, possible areas for integrating the grid structures, define the stress of the areas (stiffness/tension/pressure/bending...), lattice structure type selection by using the matrix, lattice structure implementation in the defined areas of the part, additive manufacturing of the part. The core function of the guide is the selection of a suitable grid structure as a function of the desired mechanical properties or stresses of the component.

This research was supported by the OpTi-DeP Project (no. BG101/2016) financed from the UEFISCDI by the Romanian Government and also by the HORIZON 2020 AMaTUC project (GA 691787) financed from EU.

\section{References}

1. R. Naguschewski, Stress-Based Construction of Inhomogeneous Lattice Structures and their Thermomechanical, Evaluation for Selective Laser Melting, Master Thesis (2016)

2. O. Rheme, Cellular Design for Laser Freeform Fabrication, Institute of Laser and System, (Dissertation, 2009)

3. S. J. Merkt, Qualification of generatively manufactured lattice structures for tailormade component functions, (Dissertation, 2015)

4. D. Ziebura, Development of a system for the targeted parameter determination in the SLM process using the example IN 625, (Master thesis, 2015)

5. Ch. Qiu et al., MSE, A, 638, 228-231. (2015), doi.org/10.1016/j.msea.2015.01.031

6. Ch. Yan, L. Hao, A. Hussein, P. Young and D. Raymont, Materials and Design, 55, 533-541 (2014)

7. S. McKown, Y. Shen, W.K. Brookes, C.J. Sutcliffe, W.J. Cantwell, G.S. Langdon, G.N. Nurick, M.D., Journal of Sandwich Structures \& Materials, 13(4) (2011) 